\title{
SOURCE PHENOMENOLOGY EXPERIMENTS IN ARIZONA
}

\author{
Jessie L. Bonner ${ }^{1}$, Brian Stump ${ }^{2}$, Mark Leidig ${ }^{1}$, Xiaoning Yang ${ }^{3}$, Rongmao Zhou ${ }^{2}$, Tae Sung Kim², \\ William R. Walter ${ }^{4}$, Aaron Velasco ${ }^{5}$, Chris Hayward ${ }^{2}$, Diane Baker ${ }^{3}$, C. L. Edwards ${ }^{3}$, Steven Harder ${ }^{5}$, Heather \\ Hooper ${ }^{1}$, Travis Glenn ${ }^{2}$, Cleat Zeiler ${ }^{5}$, Rengin Gok $^{4}$, James Britton ${ }^{1}$, and James F. Lewkowicz ${ }^{1}$ \\ Weston Geophysical Corporation ${ }^{1}$, Southern Methodist University ${ }^{2}$, Los Alamos National Laboratory ${ }^{3}$, Lawrence \\ Livermore National Laboratory ${ }^{4}$, and University of Texas at El Paso ${ }^{5}$ \\ Sponsored by National Nuclear Security Administration \\ Office of Nonproliferation Research and Engineering \\ Office of Defense Nuclear Nonproliferation
}

Contract Nos. DE-FC03-02SF22638 ${ }^{1}, 021364^{2}$, FIA-02-048-A001 ${ }^{3}$, L-9783 $^{4}$, and 26-8210-14

\begin{abstract}
$\underline{\text { ABSTRACT }}$
The Source Phenomenology Experiments (SPE) were conducted at mines in northeastern and southeastern Arizona in the summer of 2003. The Arizona experiments have resulted in an important dataset for the nuclear monitoring community by providing data to address a number of source parameter issues. We designed, detonated, and recorded ten single-fired explosions at a copper mine in southeast Arizona and nine single-fired explosions in a northern Arizona coal mine. The single-fired explosions ranged in size from 200 to $33000 \mathrm{lbs}$. of a mixture of Ammonium Nitrate Fuel Oil (ANFO) and emulsion explosives. Delay-fired mining explosions at each mine were also recorded. The explosions were recorded by hundreds of seismic instruments deployed at local and regional distances in addition to permanent regional broadband stations.

Using this large dataset, we developed velocity models of the Black Mesa basin, Colorado Plateau, and southern Arizona for moment tensor inversion. Detailed refraction surveys were conducted at the mine test sites in order to determine the $P$ - and $S$-wave velocities of the test beds and layer thicknesses. We used local single-component geophones (Texans) and regional broadband stations to study the $P$-wave velocity structure of the upper crust. $R g$ dispersion curves were extracted from the single-component local $(<15 \mathrm{~km})$ data and we inverted the data for $S$-wave velocity structure of the test pit region and upper crust. We also performed a joint inversion of surface wave dispersion and receiver functions to constrain the structure of the middle and lower crust.

By inverting near-source broadband data, we developed time-dependent source moment tensors for several explosions detonated during the SPE. The results show that isotropic components dominate the moment tensors, especially for the contained explosions. Vented explosions have moment-tensor spectra that are more peaked than those of fully contained explosions. Moment tensors of cast blasts show a certain amount of longer-period laterarriving energy for off-diagonal components, which is responsible for most of the shear energy generated.

We examined the regional discrimination of the SPE shots relative to normal production mining explosions, natural earthquakes, and previous nuclear tests at the Nevada Test Site. The discrimination analysis includes identifying and picking the onset times of the regional phases $P n, P g$, and $L g$, making amplitude measurements in a variety of passbands, normalizing those measurements for the effects of source and path using the Magnitude and Distance Amplitude Correction (MDAC) methodology, and calculating $\mathrm{Pg} / \mathrm{Lg}$ ratios for each event. We find that the MDAC-corrected SPE shots fall in the middle of the nuclear explosion population with very little variation due to the differing shot conditions. Thus for particular discriminants at several regional stations, the SPE shots and mining explosions are good surrogates for nuclear explosions, and the differences in depth of burial and single versus multiple shot have only a small effect on the discriminant measures. We are extending this analysis to other regional stations (e.g., ANMO, TUC, WUAZ; regional profile stations) and other discriminants to more completely examine the discrimination relationship of the SPE shots to production shots, earthquakes and nuclear tests.
\end{abstract}




\section{OBJECTIVES}

We have continued research on the large dataset collected during the Source Phenomenology Experiments (SPE) in Arizona. The goal of the project is to understand the phenomenology differences that affect various types of explosions and improve the discrimination of earthquakes and chemical and nuclear explosions. In this paper, we discuss the continued work on: 1) the inversion of near-source data for source moment tensors, 2) the effects of yield and confinement on source functions, and 3 ) the development of regional discriminants. These objectives are supported by the development of detailed site, local and regional velocity models for the regions involved.

\section{RESEARCH ACCOMPLISHED}

\section{Experiment Location and Design}

In September 2003, we detonated and recorded ten single-fired explosions at a copper mine in southeastern Arizona and nine single-fired explosions in a northern Arizona coal mine. The single-fired explosions ranged in size from 200 to $33000 \mathrm{lbs}$. of a mixture of ANFO and emulsion explosives. Delay-fired mining explosions at each mine were also recorded. Additionally, we carried out detailed refraction surveys in and around the source region to define local material properties. Figure 1 shows the mine locations and the stations deployed to record the explosions.

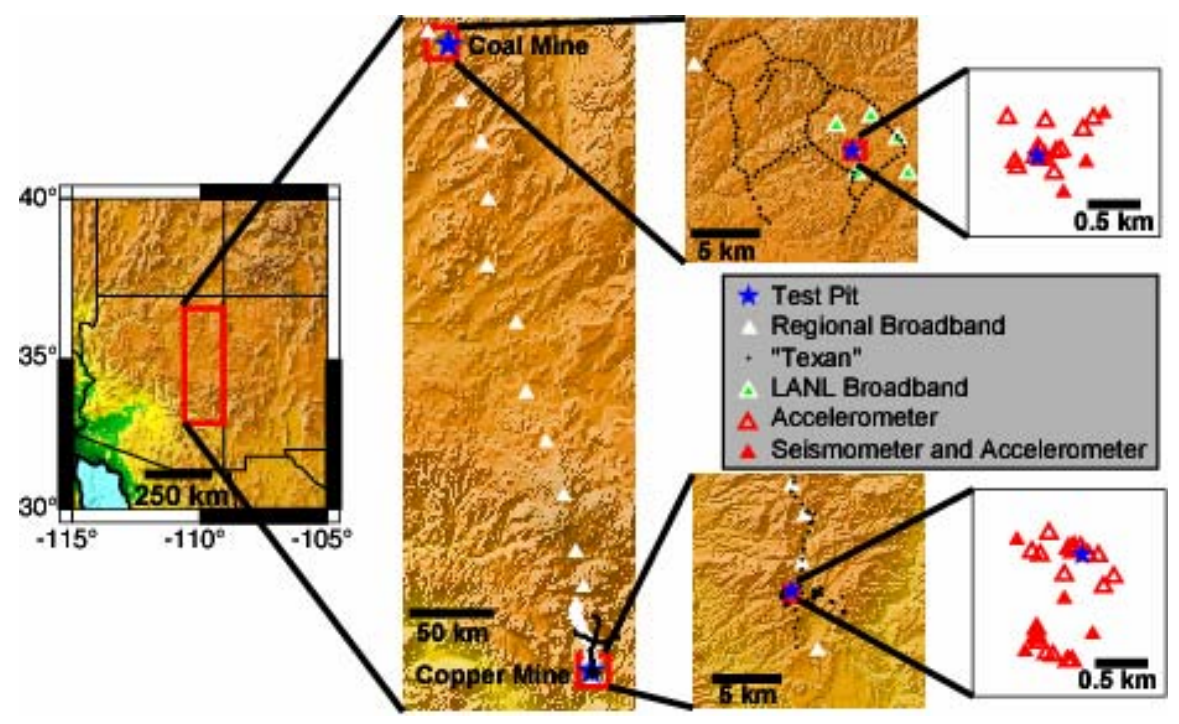

Figure 1. Location maps for the SPE mines and stations deployed.

\section{$\underline{\text { Velocity Structure }}$}

The development of a detailed velocity and attenuation structure for the coal mine in northern Arizona is discussed in the Seismic Research Review paper by Leidig et al. (2004) and also in Leidig et al. (in review). The explosion medium at the coal mine was determined to be slow and indicative of highly-fractured, dry sedimentary rock. Leidig et al. (2004) also discusses the development of a velocity model for the copper mine in southern Arizona. Below is a further discussion of the velocity model for the copper mine.

Detailed refraction surveys were conducted at the copper mine test site in order to determine the $P$-wave velocities $(\mathrm{Vp})$ of the test bed. The structure consisted of a shallow $(4-5.5 \mathrm{~m})$ weathered layer $(0.58<\mathrm{V} p<0.67 \mathrm{~km} / \mathrm{sec})$, a second layer $(\sim 18 \mathrm{~m}$ with $2.44<\mathrm{V} p<3.05 \mathrm{~km} / \mathrm{sec})$, and faster velocities at greater depths $(\mathrm{V} p>4.57 \mathrm{~km} / \mathrm{sec})$. The refraction studies are described in detail in Hayward et al. (2004). The velocities of the emplacement media in this granite porphyry are significantly faster than the limestone test pit at the coal mine. 
The compressional-wave velocity was further constrained using the first arrivals of $P$ - waves recorded on a northsouth line of Texans north of the copper mine. A $P$-wave velocity structure of two layers over a halfspace, with velocities estimated to be $4.55,5.21$, and $6.07 \mathrm{~km} / \mathrm{s}$ respectively, was inferred from the travel-time curves. The corresponding layer thicknesses were calculated to be $0.15 \mathrm{~km}$ and $3.38 \mathrm{~km}$, using the relationship between velocity, critical distance, and intercept time (Dobrin, 1960).

The dispersion curves of the $\mathrm{Rg}$ phase recorded on the N-S broadband station profile were used to provide shear wave velocity constraints for our model. The dispersion curves show a systematic variation, implying the existence of a strong lateral variation in the velocity structure around the mine (Figure 2a). It is believed that the variation is caused by a change from a granodiorite body (faster dispersion curves) located in the source region $(0-7 \mathrm{~km}$ in Figure $2 \mathrm{~b}$ ) to deformed sedimentary rocks (slower dispersion curves). The velocity models produced from differential inversion of these dispersion curves are tabulated in Kim and Stump (in preparation).
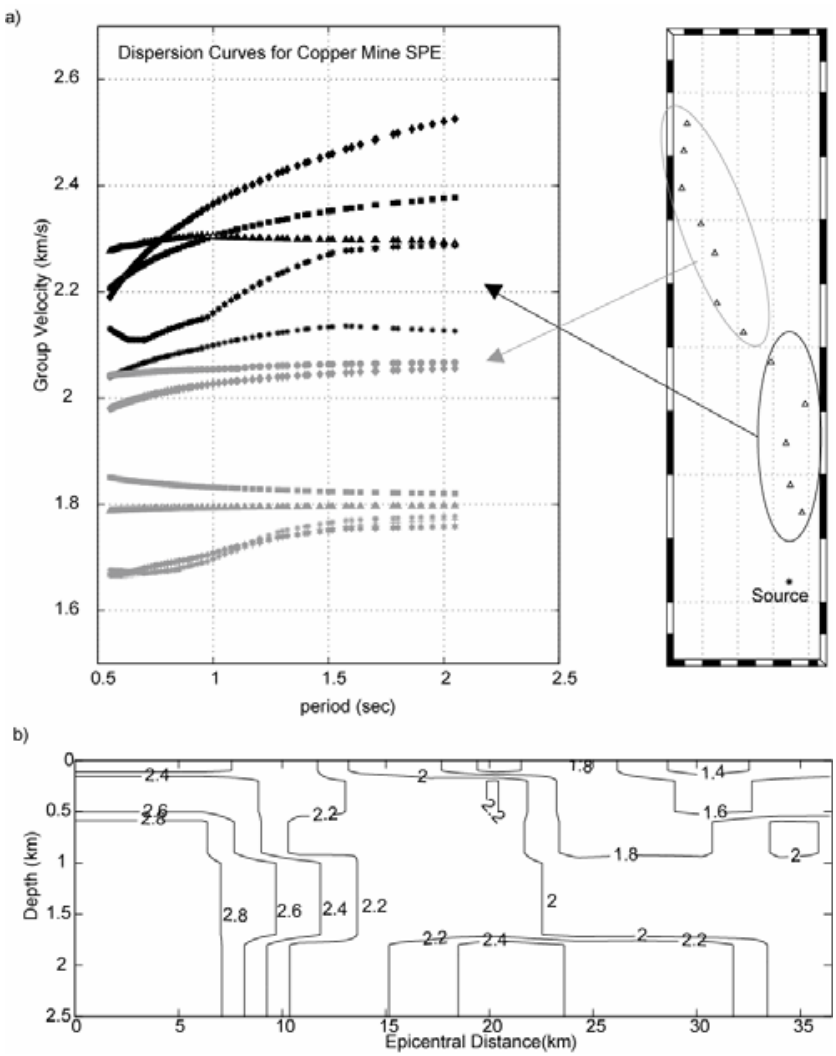

Figure 2. Shear wave velocity data for the source region at the copper mine. a) Short-period, fundamentalmode Rayleigh wave $(\mathrm{Rg})$ group velocity dispersion curves from broadband recordings showing possible lateral variations in shear-wave velocity structure. b) Differential inversions of the dispersion curves showing changes in the structure with increasing distance from the sources.

\section{Moment Tensor Inversion}

We used the Leidig et al. (in review) velocity model from northern Arizona in moment tensor inversions of shots 3 (fully contained), 4 (partially contained), and 9 (uncontained cast shot without delays between holes). The momentrate tensors all show strong isotropic characteristics (Figure 3). The acausal appearance of the shot 9 moment tensors was possibly caused partly by the point-source approximation that is not as accurate for cast blasts as for contained shots. Further information can be extracted from the moment-rate tensor spectra in Figure 4. Although shots 3 and 4 were of about the same size, their diagonal moment-tensor component spectra are not quite the same. Shot 3 (Figure 4, left) has larger amplitudes (a factor of about 1.7) and lower corner frequency than that of shot 4 (Figure 4, center; $13 \mathrm{~Hz}$ vs. $16 \mathrm{~Hz}$ ). Spectra of diagonal moment-tensor components of shot 4 are more peaked, possibly due to stronger spall. For shot 9 (Figure 4, right), a considerable amount of Myz energy is seen in the frequency band between 1 and $6 \mathrm{~Hz}$. There is also enhanced Mzz energy in this frequency band. Figure 5 shows the bandpass 
filtered $(1-6 \mathrm{~Hz})$ moment-rate tensors of shots 3 and 9. There is a clear signal enhancement in the Myz component of shot 9 (Figure 5, right) compared with shot 3 (Figure 5, left). The Myz signal is lower frequency than that of the initial pulse and may also be delayed in time. This could indicate that the signal was generated by the bench material cast into the pit.

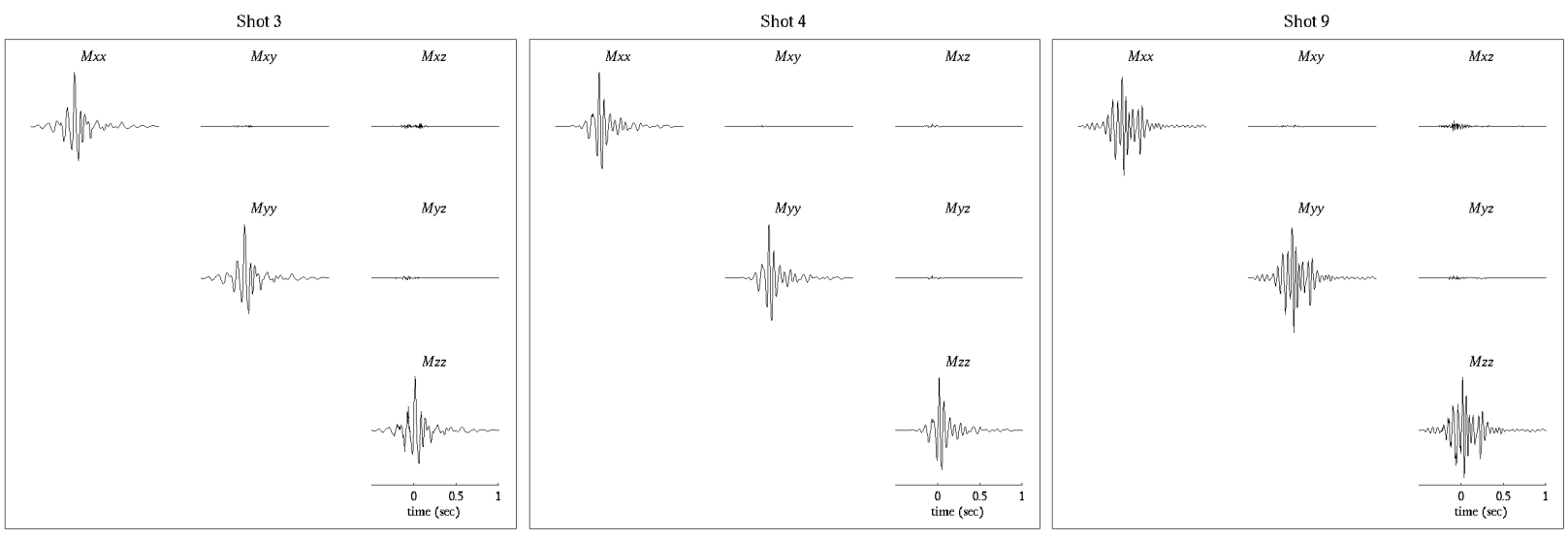

Figure 3. Moment tensor inversion results for shot 3 (left), shot 4 (center), and shot 9 (right).
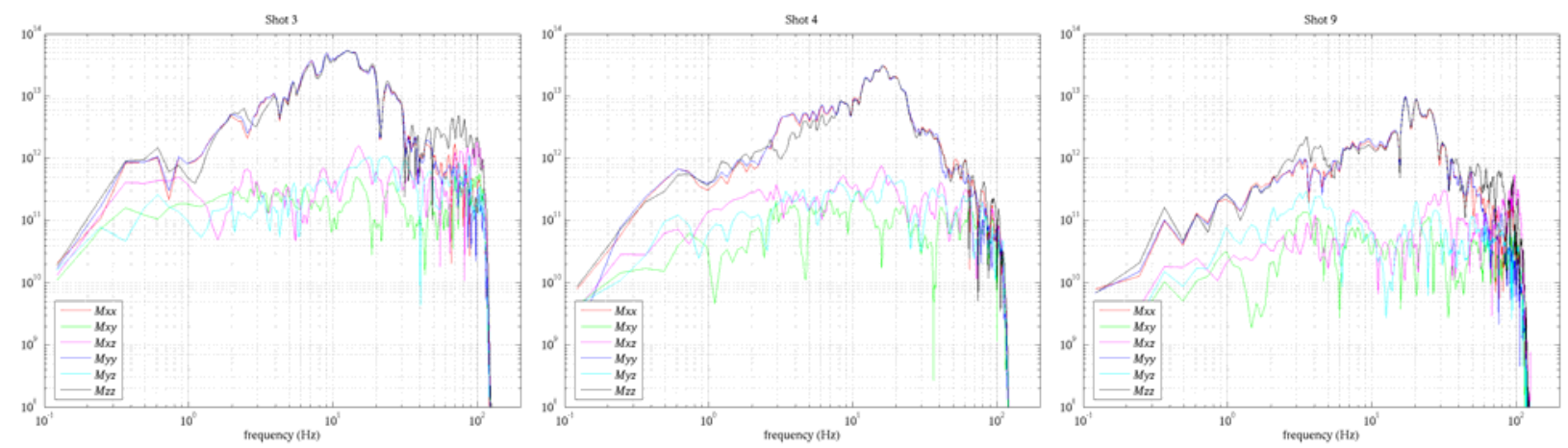

Figure 4. Moment-rate tensor spectra for shot 3 (left), shot 4 (center), and shot 9 (right).
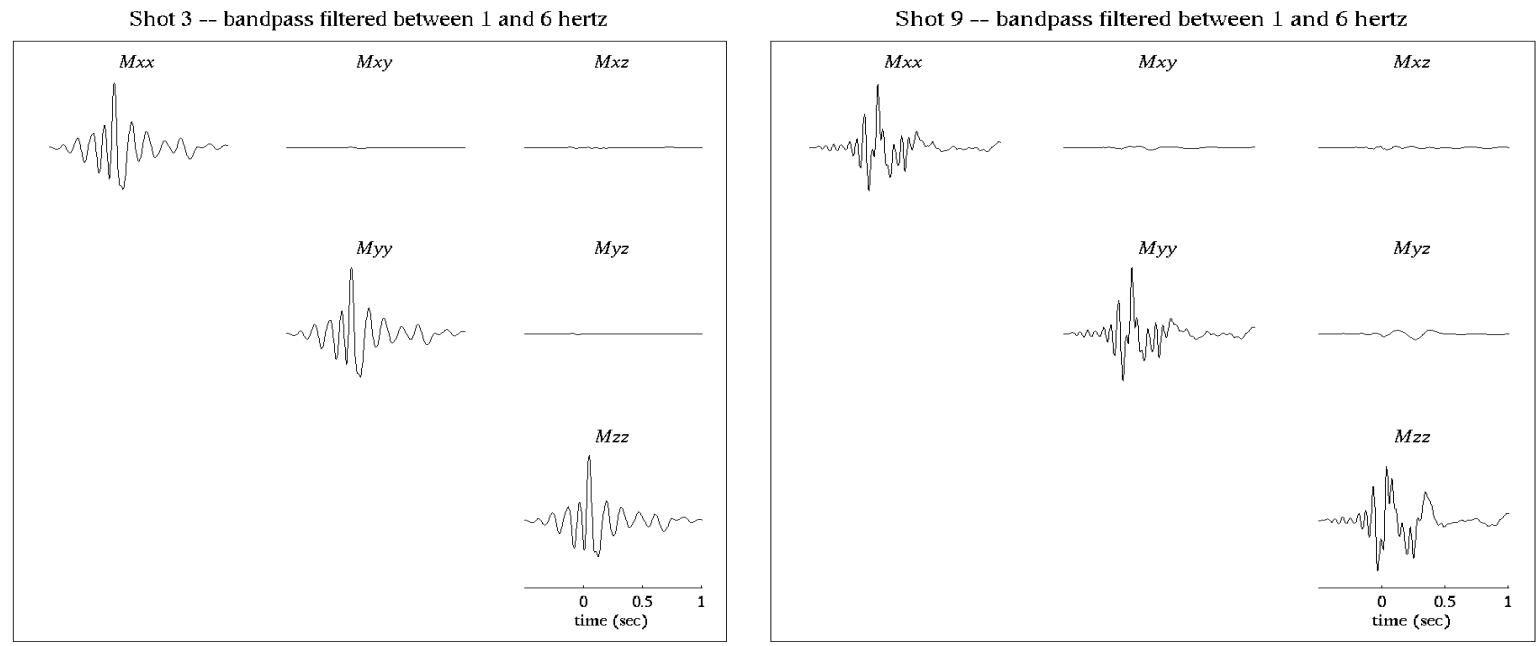

Figure 5. Moment tensor inversion results for shot 3 (left) and shot 9 (right), both bandpass filtered between 1 and $6 \mathrm{~Hz}$. 
Although the amplitude of the Myz component of shot 9 is only about $7 \%$ of the diagonal components, it can generate a much larger percentage of shear energy. Figure 6 plots synthetic seismograms calculated using the shot 9 source at an azimuth of zero degrees and a distance of approximately $5 \mathrm{~km}$. The transverse component amplitude generated by the off-diagonal moment tensor is about $30 \%$ of the $P$-wave amplitude generated by the explosive source.

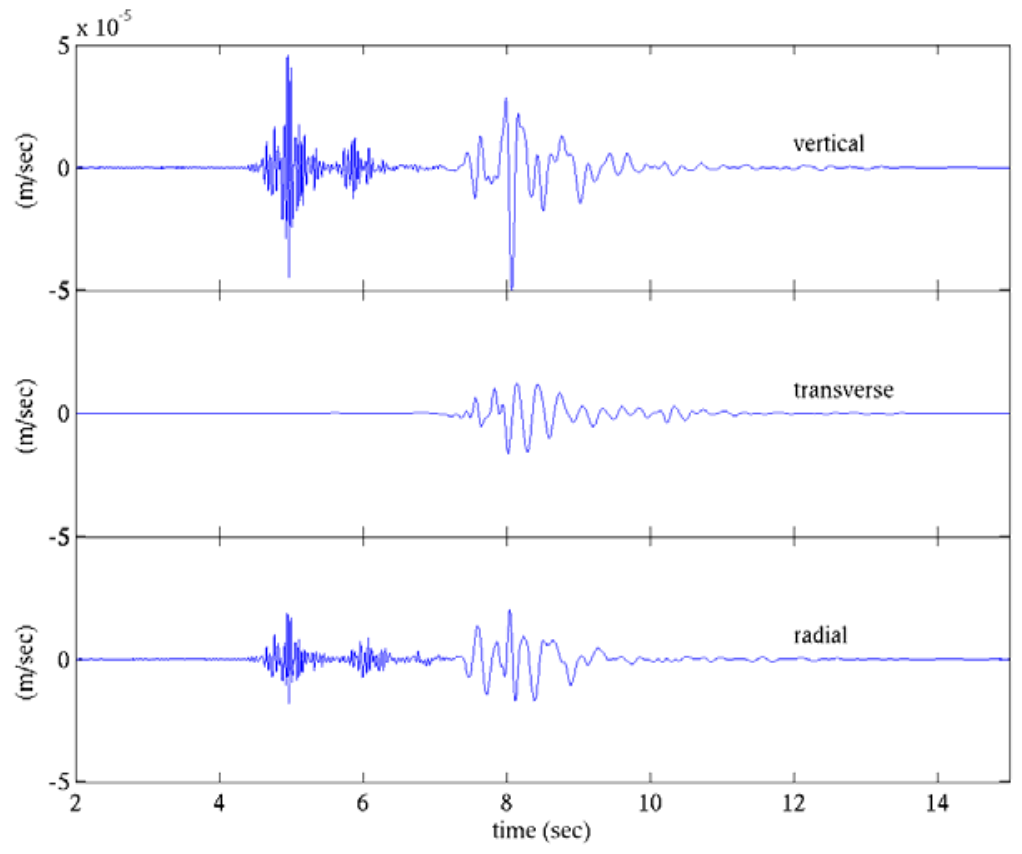

Figure 6. Synthetic seismograms based on the moment tensors for shot 9.

\section{Effects of Yield and Confinement}

Research on the effects of yield and confinement at the copper mine was initially reported on by Leidig et al. (2004). The following provides an update to this research at the southern Arizona copper mine. In Figure 7, the effects of yield are quantified at various station distances by comparing the spectral ratios for shots with the same confinement and different explosive size. In general, the spectral ratios at all distance ranges are similar in shape and amplitude indicating a common source scaling with distance.

The effects of confinement have been studied by comparing the spectral ratios for shots with identical yield and the results are presented in Figure 8. The spectral ratios of twice depth to free face for 13600 and $1700 \mathrm{lbs}$. shots are relatively flat at all frequencies. They indicate a reduction of coupling on the free face shots of $\sim 2-4$. Similar reduced coupling is also found by comparing the data from the twice burden and free face shots. These results suggest that the failure of the free face results in a reduction in seismic energy at all distances ranges.

Source modeling. Figure 9 presents the comparison of the predicted spectral ratios from the Mueller-Murphy granite model source function (dotted lines) with the shots of twice depth (Figure 7a). The $P$-wave velocity model $(\mathrm{V} p=3.048 \mathrm{~km} / \mathrm{s})$ of the source materials was based on the in-mine refraction survey assuming Poisson medium for shear wave velocity and density $=2.2 \mathrm{gm} / \mathrm{cc}$. The model matches the observed data to $6 \mathrm{~Hz}$, above which the observed ratios begin to increase. 
27th Seismic Research Review: Ground-Based Nuclear Explosion Monitoring Technologies

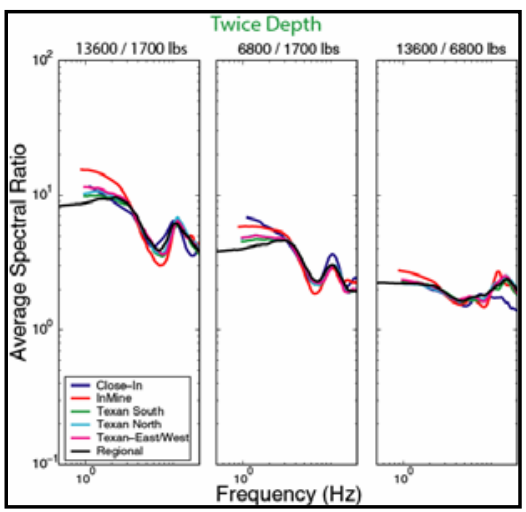

(a)

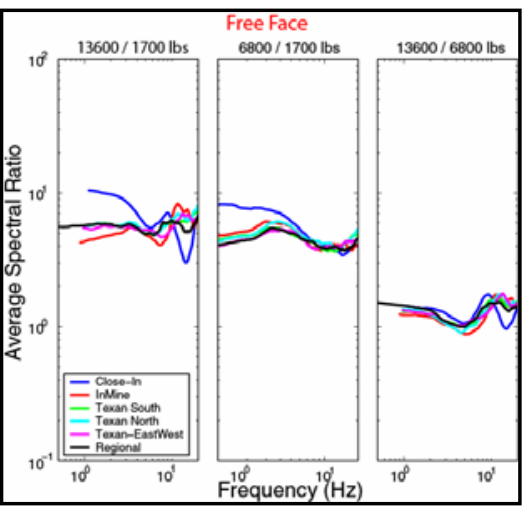

(b)

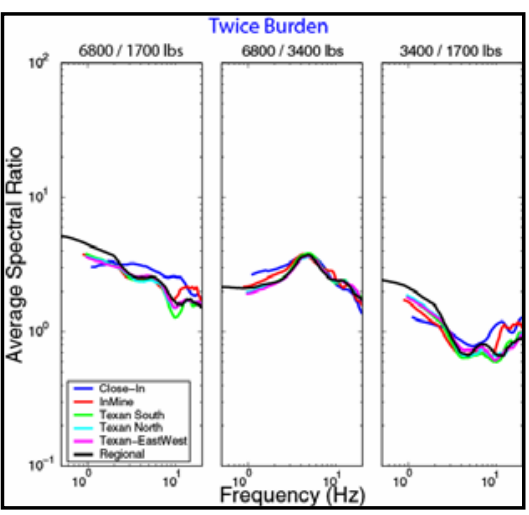

(c)

Figure 7. Spectral ratios of shots with same confinement and different yield, (a) twice depth; (b) free-face; and (c) twice burden.

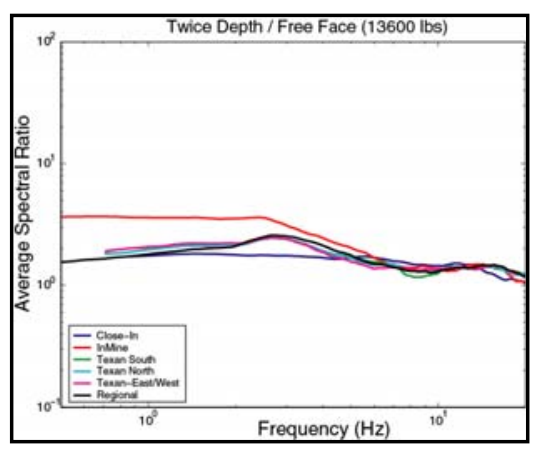

(a)

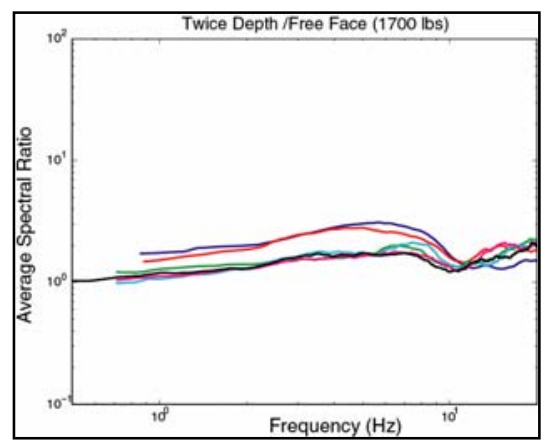

(b)

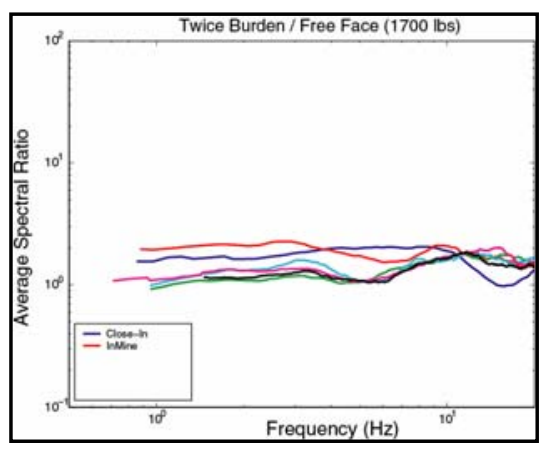

(c)

Figure 8. Spectral ratios of shots with same explosive size and different confinements, (a): twice depth / free face (13600 lbs); (b) twice depth / free face (1700 lbs); and (c) twice burden / free face (1700 lbs).
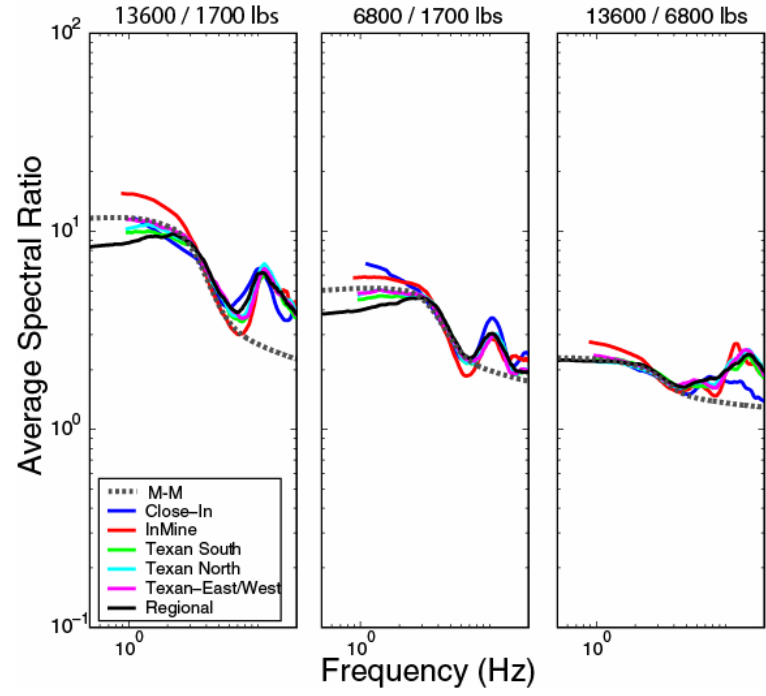

Figure 9. Comparison of the predicted spectral ratios from the Mueller-Murphy granite model (dotted line) with twice depth shots. 
Effects of Confinement on Short-Period Surface Waves. Hooper et al. (2004) reported research on the excitation of short-period, fundamental mode Rayleigh waves $(R g)$ by explosions, using SPE data collected from the network of vertical component geophones. This research has continued, and we have explored the effects of confinement on the generation of $R g$ from explosions.

Results show important differences between the $R g$ amplitudes of confined and unconfined explosions that need to be understood in order to develop discriminants for mining explosions. $R g$ energy and frequency content are dependent upon explosive weight and confinement, and unconfined explosions generate less energy than equivalent confined explosions due to decoupling effects. We calculated empirical decoupling factors, which provide a firstorder estimate of the frequency-dependent decoupling caused by the lack of confinement. The decoupling factors are calculated by taking the ratios of observed and theoretical spectral ratios from the explosions:

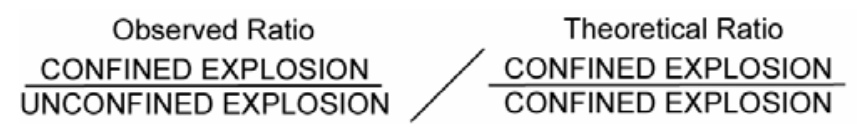

The observed confined explosion is shot 3, and shots 4-9 are unconfined. The theoretical spectral ratios are based on Mueller-Murphy sources (all confined) simulating the same shots.

The chemical decoupling factors for unconfined shots vary from 1 to 10 at frequencies between $0.5 \mathrm{and} 8 \mathrm{~Hz}$, indicating that unconfined explosions generate up to 10 times less $R g$ energy than confined explosions (Figure 10). For this reason, unconfined mining explosions cannot be simulated using a Mueller-Murphy (1971) source without including an empirical chemical decoupling factor such as those described above. Additionally, studies of a small, overburied shot indicate that some $\mathrm{Rg}$ excitation effects are not being adequately modeled by the Mueller-Murphy source and the available medium-dependent properties (Hooper et al., in review).

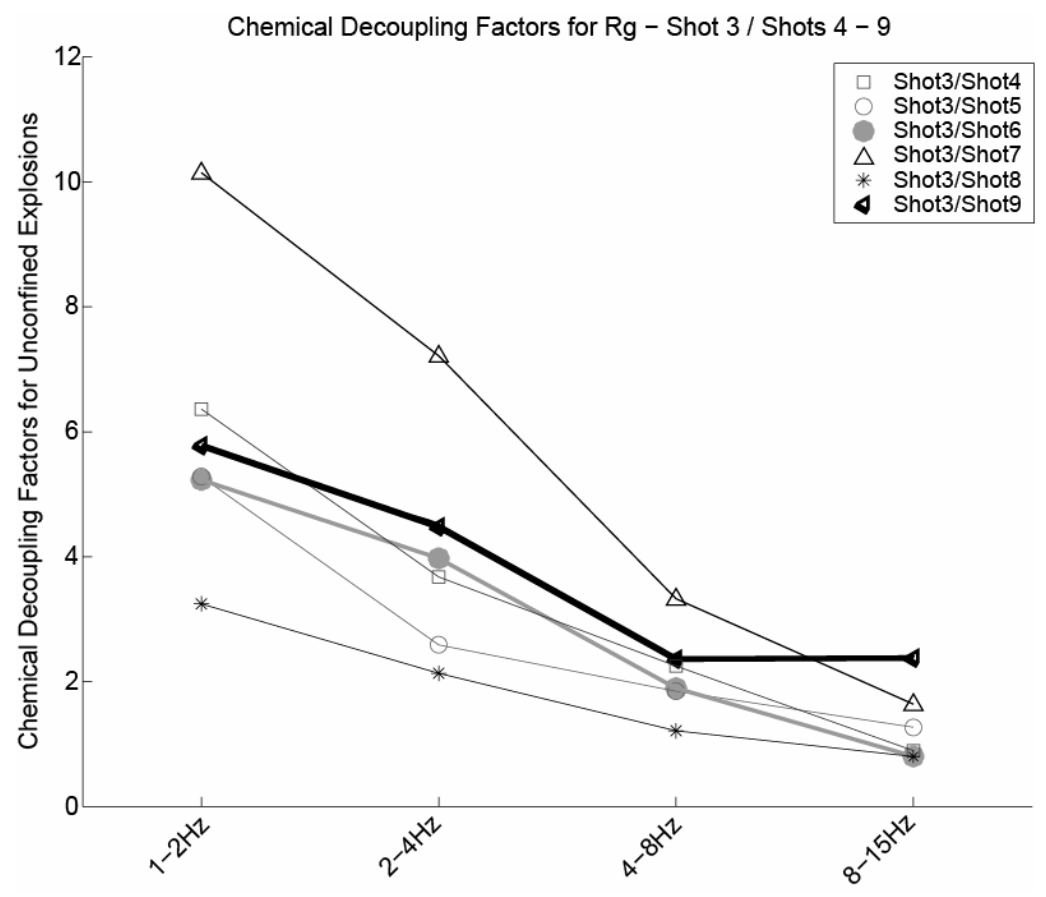

Figure 10. Chemical decoupling factors (observed ratio / theoretical ratio) calculated for the shot 3 (confined) / shots 4-9 (unconfined) ratios for $\boldsymbol{R g}$ for various frequency bands. These chemical decoupling factors are mainly due to the differences between the unconfined and confined sources. 


\section{$\underline{\text { Regional Discrimination }}$}

We have started to examine the regional discrimination behavior of the SPE shots relative to normal production mining explosions, natural earthquakes, and previous nuclear tests at the Nevada Test Site. Examples of the regional seismograms from the northern Arizona coal mine SPE shot 3 are shown in Figure 11. The discrimination analysis includes identifying and picking the onset times of the regional phases $\mathrm{Pn}, \mathrm{Pg}$, and $\mathrm{Lg}$, making amplitude measurements in a variety of passbands, normalizing those measurements for the effects of source and path using the Magnitude, Distance, and Amplitude Correction (MDAC) methodology (Walter and Taylor, 2002), and calculating $\mathrm{Pg} / \mathrm{Lg}$ ratios for each event. Figure 12 shows the results at station KNB (Kanab, Utah) for six of the northern Arizona coal mine SPE shots (diamonds) and production mining explosions (triangles), compared with a set of nuclear tests (stars) and western U.S. earthquakes (circles). We find that the MDAC-corrected SPE shots fall in the middle of the nuclear explosion population with very little variation due to the differing shot conditions. Thus for this particular discriminant at this station, the SPE shots and mining explosions are good surrogates for nuclear explosions, and the differences in depth of burial and single versus multiple shot have only a small effect on the discriminant measures. We are extending this analysis to other regional stations (e.g., ANMO, TUC, WUAZ; regional profile stations) and other discriminants to more completely examine the discrimination relationship of the SPE shots to production shots, earthquakes and nuclear tests.

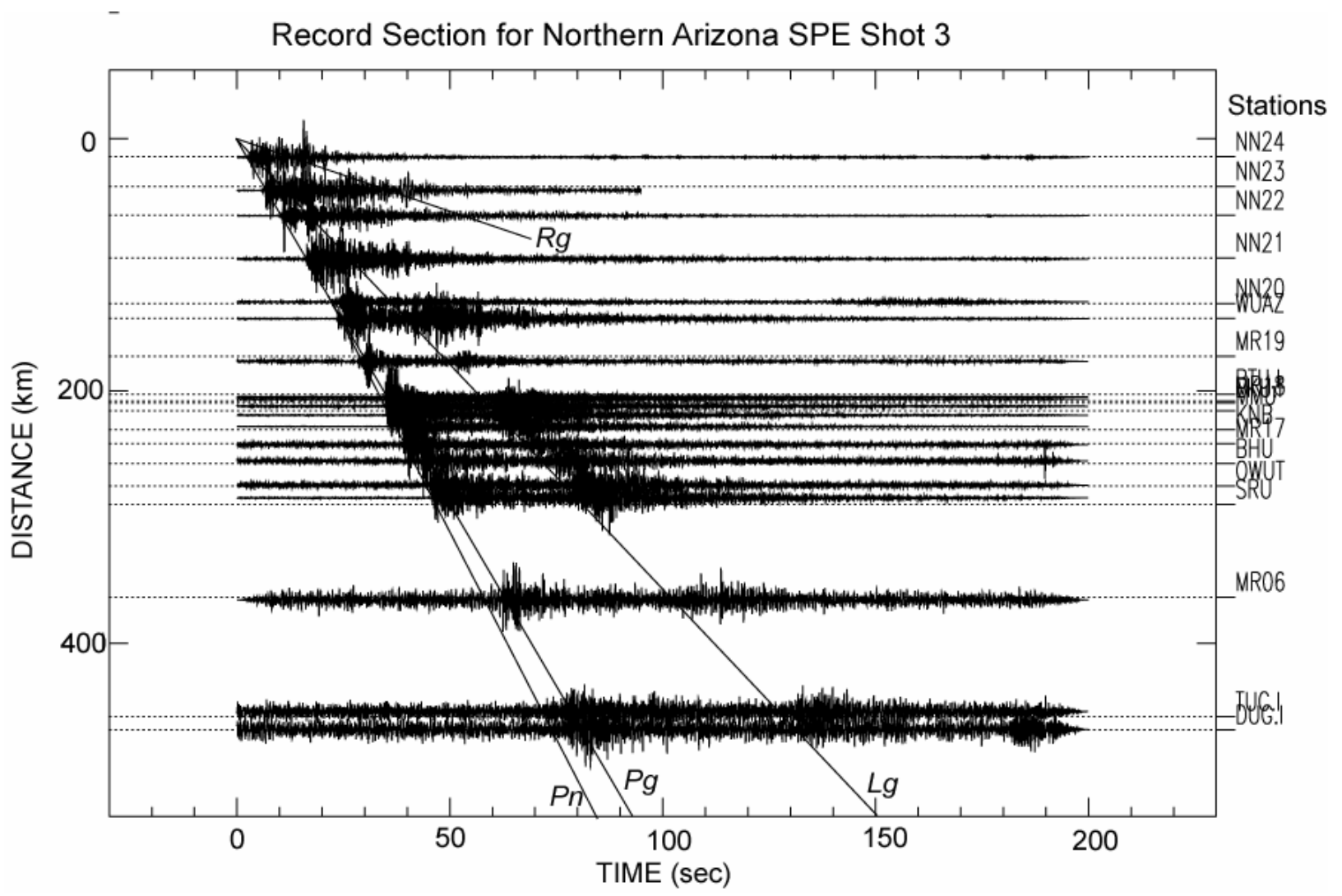

Figure 11. Record section of near-regional data recorded from SPE shot 3 at the coal mine in northern Arizona. $P n, P g$, and $L g$ are the prominent arrivals at regional distances. At local distances, we note a prominent $1 \mathrm{~Hz} \mathrm{Rg}$ arrival; however, it is highly attenuated in the upper crust of the Colorado Plateau. 

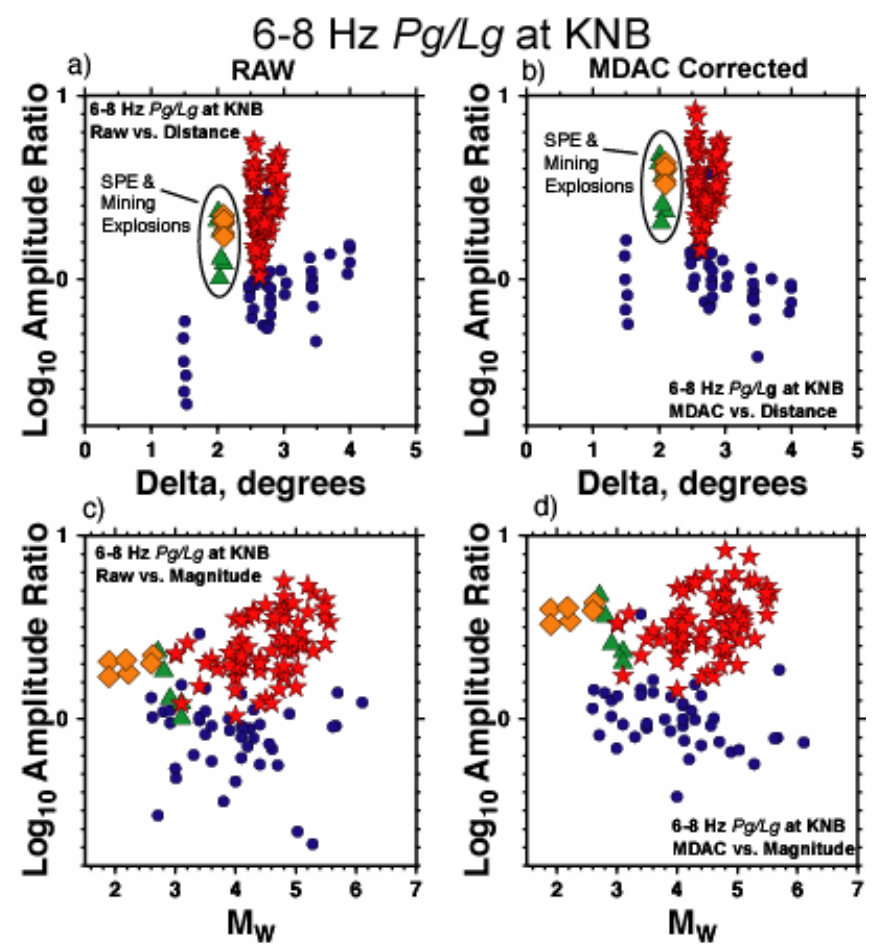

Figure 12. Comparison of six SPE shots from the northern Arizona coal mine (diamonds) with production shots at the same mine (triangles) at station KNB using the 6-8 $\mathrm{Hz} \mathrm{Pg} / \mathrm{Lg}$ discriminant (e.g. Walter et al., 1995). The ratios are plotted as either raw (a and c) or MDAC-corrected (b and d) amplitude ratios as a function of distance (a and b) or moment magnitude (c and d). The SPE shots show less variability and have similar values to the mean of the nuclear tests (stars), making them possible surrogates in this discriminant case. Earthquakes are plotted as circles.

Regional coda envelopes provide very stable single station estimates of source spectra (e.g., Mayeda et al. 2003). Here we have calibrated the paths based on Colorado Plateau earthquakes. For each station we can then examine the differences in source spectra as determined from the coda (Figure 13). The differences in source spectral shape due to depth and confinement are quite subtle. Between the two mines the hard rock copper site shows less 6-8 Hz energy than the coal mine. This is clear in the comparison of similar size (12,000 and 13,600 lbs) and depth (30 and $33 \mathrm{~m}$ ) events. This difference causes the copper mine to have larger low/high spectral-ratios discriminant values than we observe for the coal mine.
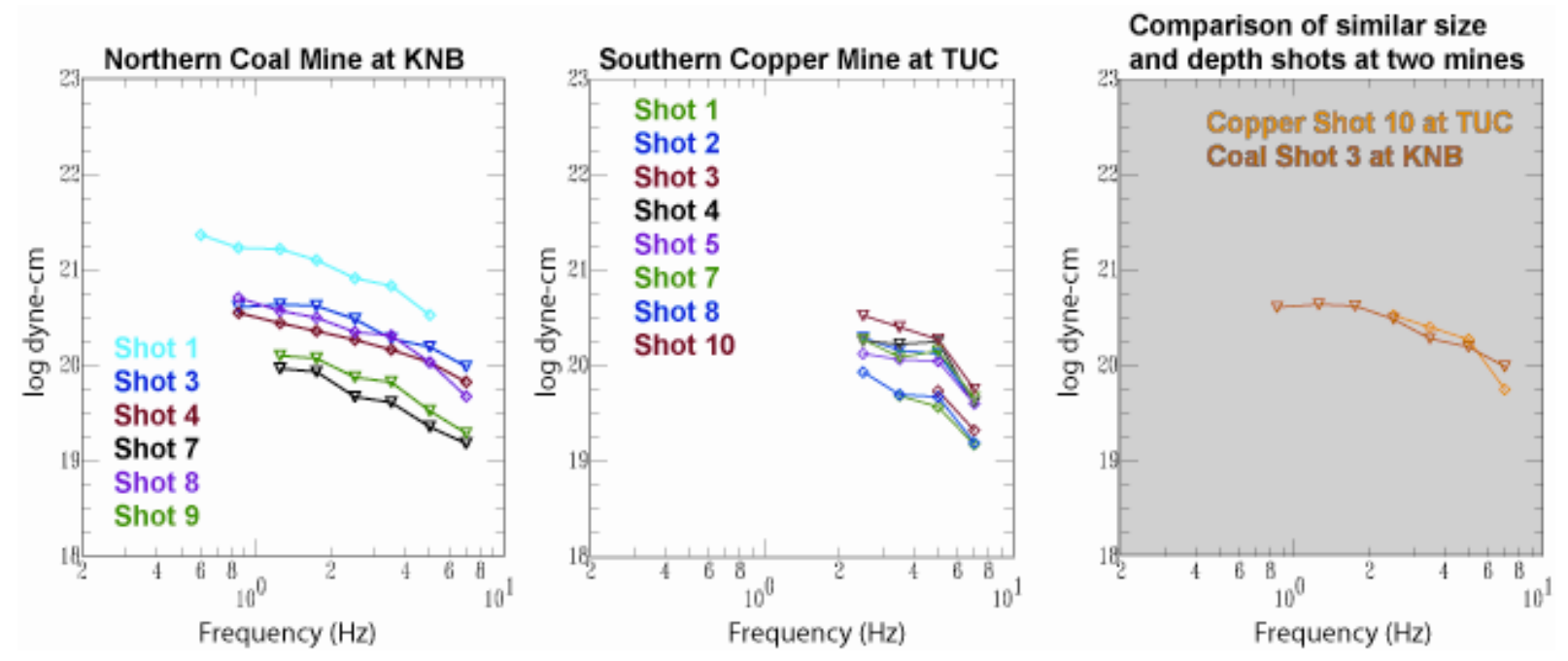

Figure 13. Source spectra for shots at the northern Arizona coal mine and the southern Arizona copper mine. 


\section{CONCLUSIONS AND RECOMMENDATIONS}

The Arizona SPE experiments have resulted in an important dataset for the nuclear monitoring community. The 19 dedicated single-fired explosions and multiple delay-fired mining explosions were recorded by one of the most densely instrumented accelerometer and seismometer arrays ever fielded, and the data have already proven useful in quantifying confinement and excitation effects for the sources. It is very interesting to note that we have observed differences in the phenomenology of these two series of explosions resulting from the differences between the relatively slow (limestone) and fast (granodiorite) media. We observed differences at the two SPE sites in the way the rock failed during the explosions, how the $S$-waves were generated, and the amplitude behavior as a function of confinement. Our consortium's goal is to use the synergy of the multiple datasets collected during this experiment to unravel the phenomenological differences between the two emplacement media. The results so far suggest that the single-fired explosions are surrogates for nuclear explosions in higher frequency bands (e.g., 6-8 Hz Pg/Lg discriminants).

\section{ACKNOWLEDGEMENTS}

We express our sincere thanks to all involved and to those who helped support the Source Phenomenology Experiments. This research would not have been possible without the tremendous support of the front office, engineers, and miners of the mines used in this study. We slowed their production schedules, yet they were always gracious and wonderful hosts. We thank Darlene McEwand, John Sandru, Aaron Hirsch, and the University of Nevada Las Vegas for teaching us how to bury a Texan. We thank the Navajo Nation for allowing us to place instruments on their lands. In addition, we thank IRIS PASSCAL for their help on this project. We also wish to thank Mr. Mike Rosenthal for his support in obtaining variances for the largest explosions.

\section{REFERENCES}

Dobrin, M. B., (1960), Introduction to Geophysical Prospecting. 2nd edition, McGraw-Hill Book Co, Inc., New York.

Hayward, C., R.-M. Zhou and B. Stump, (2004), QuickLook Report: 2004 January Morenci Refraction Experiments and Morenci Refraction Interpretation, February 2004, Southern Methodist University.

Hooper, H., H. Patton, J. Bonner, and M. Leidig, (2004), Using short-period surface waves to model source phenomena, in 26th Seismic Research Review: Trends in Nuclear Explosion Monitoring, LA-UR-04-5801, Vol. 1, pp. 417-426.

Hooper, H., J. L. Bonner, and M. Leidig, (in review), Effects of confinement on short-period surface waves: Observations from a new dataset. Submitted to Bull Seism. Soc. Am.

Kim, T.S. and B.S. Stump (in preparation). Upper crustal velocity structure around the Morenci copper mine from $\mathrm{Pg}$ and $\mathrm{Rg}$ phases.

Leidig, M., J. Bonner, and D. Reiter, (in review), Development of a velocity model for Black Mesa, Arizona and the southern Colorado Plateau from multiple datasets, Submitted to the Bull. Seism. Soc. Am.

Leidig, M., B. Stump, J. Bonner, C. Hayward, A. Velasco, D. Baker, H. Hooper, W. Walter, X. Yang, R. Zhou, C.L. Edwards, M. Renwald, and J. Lewkowicz (2004). Source phenomenology experiments in Arizona, Proceedings of the 26th Seismic Research Review: Trends in Nuclear Explosion Monitoring, LA-UR-04-5801, Vol. 1, pp. 427-438.

Mayeda, K., A. Hofstetter, J.L. O'Boyle, and W.R. Walter (2003), Stable and transportable regional magnitudes based on coda-derived moment-rate spectra, Bull. Seism. Soc. Am. 93: 224-239.

Mueller, R.A., and J.R. Murphy, (1971), Seismic characteristics of underground nuclear detonations. Bull. Seism. Soc. Am., 61: 1675-1692.

Walter, W.R., K.M. Mayeda, and H.J. Patton, (1995), Phase and spectral ratio discrimination between NTS earthquakes and explosions, Part 1, Empirical Observations, Bull. Seism. Soc. Am. 85: 1050.

Walter, W. R. and S. R. Taylor (2002), A revised magnitude and distance amplitude correction (MDAC2) procedure for regional seismic discriminants. Lawrence Livermore National Laboratory UCRL-ID-146882. 